Z. Phys. D (Proceedings of the Sixth International Conference on Small Particles and Inorganic Clusters, Sept. 16-22, 1992, Chicago, Illinois), NP, IT

\title{
Cluster-molecule systems: Analysis and tuning of the interaction potential
}

\section{L.Jellinek and Z. B. Güvenç}

Chemistry Division, Argonne National Laboratory, Argonne, IL 60439, USA

\section{DISCLAIMER}

\begin{abstract}
This report was prepared as an account of work sponsored by an agency of the United States Government. Neither the United States Government nor any agency thereof, nor any of their employees, makes any warranty, express or implied, or assumes any legal liability or responsibility for the accuracy, completeness, or usefulness of any information, apparatus, product, or process disclosed, or represents that its use would not infringe privately owned rights. Reference herein to any specific commercial product, process, or service by trade name, trademark, manufacturer, or otherwise does not necessarily constitute or imply its endorsement, recommendation, or favoring by the United States Government or any agency thereof. The views and opinions of authors expressed herein do not necessarily state or reflect those of the United States Government or any agency thereof.
\end{abstract}




\title{
Cluster-molecule systems: Analysis and tuning of the interaction potential
}

\author{
J. Jellinek and Z. B. Güvenç \\ Chemistry Division, Argonne National Laboratory, Argonne, Il 60439, USA
}

Received 16 September 1992

\begin{abstract}
The interaction (modelled by a LEPS potential) of a $\mathrm{D}_{2}$ molecule with an icosahedral $\mathrm{Ni}_{13}$ cluster is mapped and analyzed in the form of equipotential contour plots. The topological features of the map correlate with the energetic and dynamical characteristics of the dissociative adsorption of the molecule on the cluster, which were extracted from quasiclassical trajectory simulations. A modification of the LEPS potential used originally is introduced. It brings the calculated reactivity of the cluster into agreement with the experimental data.
\end{abstract}

PACS: $34.10 .+\mathrm{x} ; 36.40 .+\mathrm{d} ; 82.20 . \mathrm{Fd} ; 82.20$. Wf

\section{Introduction}

An intriguing and challenging area of the field of atomic and molecular clusters and cluster-related phenomena is that of interactions (collisions) of clusters with molecules. These interactions are expected to (and there are indications that they do indeed) possess features similar to those of the much more extensively studied interactions of molecules with molecules, on the one hand, and molecules with surfaces, on the other. At the same time, the phenomena induced by molecule-cluster collisions may bj, and usually are, more intricate because typically a larger number of degrees of freedom is involved.

Interactions of metal clusters with molecules are of especial interest because of their relevance to a variety of technologically important processes. These interactions have been studied experimentally in the last few years quite intensively [1]. In the majority of theoretical studies, electronic structure calculations were performed to determine tnergetically preferred configurations of cluster-molecule systems [2]. Often the clusters were prepared as slabs of lattices to model surfaces of the bulk material [3]. Only in a

\footnotetext{
Work performed under the auspices of the Office of Basic Energy Sciences, Division of Chemical Science, US-DOE under contract number W-31-109-ENG-38.
}

few theoretical treatments have the cluster-molecule interactions been studied as dynamical processes [4-10].

We have carried out extensive quasiclassical trajectory simulations of a collision of a $\mathrm{D}_{2}$ molecule with a $\mathrm{Ni}_{13}$ cluster. The cluster was modelled by an embedded-atom potential of Voter and Chen [11], the parameterization of which takes into account properties of both the bulk nickel and the $\mathrm{Ni}_{2}$ molecule. The D-D and the molecule-cluster interactions were originally represented by a LEPS (London-Eyring-Polanyi-Sato) function as parameterized by Raghavan, Stave and DePristo (RSD) [4]. More specifically, we have utilized the so-called potential energy surface (PES)II of RSD, which was adjusted to represent the interaction of a $D_{2}$ with a $\mathrm{Ni}_{13}$, in contrast to the PESI, which was fitted to model the interaction of the molecule with a surface of the bulk nickel. We have slightly modified the PESII by a smoothing function of the same form as used by Truong et al [12].

All the possible outcomes of the collision - inelastic scattering of the molecule from the cluster (nonreactive) and dissociative adsorption of the molecule on the cluster (reactive) - were considered and characterized as functions of the initial (and, in the case of inelastic scattering, also final) quantized rovibrational state of $D_{2}$, impact parameter, collision energy, and structire and temperature of the cluster. The quantitative analysis was performed in terms of detailed probabilities, cross sections, rate constants and activation energies. Partial accounts of the results can be found in Refs. 6-8, 10. The major findings can be summarized as follows: (1) overall, the propensity for the dissociativi adsorption increases with an increase of the initial rovibrational energy of $D_{2}$ and/or of the system collision energy; (2) the reaction is mode-selective: an initial vibrational excitation of the molecule results in larger cross sections of dissociative adsorption than an energetically equal initial rotational excitation; (3) the reactivity of the cluster depends on its structure: the most stable icosahedral (ico) form of $\mathrm{Ni}_{13}$ is less reactive than its hexagonal closepacked (hcp) and cuboctahedral (cubo) isomers. The former 
exhibits energy thresholds to the reaction, while the latter do not; (4) the reactivity of the cluster does not change when its temperature is varied from $\mathrm{T}=0 \mathrm{~K}$ to $\mathrm{T}=300 \mathrm{~K}$ and increases only moderately when the temperature is raised up to $\mathrm{T}=1000 \mathrm{~K}$; (5) both the reaction and the inelastic scattering of the molecule can be direct or indirect. The indirect processes involve transient states of molecular adsorption (classical) resonances. The structure-reactivity correlation exhibits itself also in that, at very low collision energies, $D_{2}$ forms reactive resonances (i.e., those which eventually dissociate on the cluster) with the hcp and cubo forms of $\mathrm{Ni}_{13}$, but not with its ico isomer. As a consequence, the reaction cross sections for the hcp and cubo geometries possess (local) maxima at low collision energies. We have introduced a new resonance analysis technique and applied it to calculate the probabilities (cross sections) of formation of reactive resonances and their lifetimes $[8,10,13]$. The lifetimes and the relative contribution of the resonances to the overall reactivity decrease with increase of the collision energy.

Although the experimental data available at present do not allow for detailed verification of the predictions listed above, recent measurements on reactivity of nickel clusters indeed indicate only a weak temperature-dependence and a possible involvement of resonances [14]. However, the calculated room-temperature rate constant of the reaction $\mathrm{Ni}_{13}$ $+\mathrm{D}_{2} \rightarrow \mathrm{Ni}_{13} \cdot 2 \mathrm{D}$ [10] (cf. also Ref. 4) disagrees with the value deduced from the experiments [15]. A factor that plays a dominant role in defining the qualitative and quantitative characteristics of the reaction is the topology of the cluster-molecule potential energy surface. The goal of this communication is to present an analysis of the LEPS surface that leads to the results mentioned above. This is done in the next section. In Sect. 3 we consider a modification of the LEPS that brings the calculated reaction rate constant into an agreement with the value obtained from the measurements. A summary is given in Sect. 4.

\section{Analysis of the topology of the LEPS surface}

A useful way of displaying the features of a potential energy surface, which allows one to literally see the "wells" and "barriers" ("saddles"), is mapping it in the form of equipotential contour plots (ECP). These plots are widely used in analyses of atom-diatom and diatom-surface interactions. An inherent constraint of the ECPs is that they are restricted to two independent variables at a time. This, however, usually is not a real limitation.

As mentioned, we have used the PESII parameterization of RSD [4] in the LEPS representation of the cluster-molecule interaction. Our considerations here are restricted to the ico form of $\mathrm{Ni}_{13}$. By carrying out complete dynarnical quenchings, we have found that the adsorption of a $D_{2}$ (either molecular or dissociative) causes only a negligible perturbation in the cluster structure (the energy of the equilibrium ico configuration changes by less than $0.01 \%$ ). This justifies considering the cluster degrees of freedom as frozen. The cluster-molecule interaction energy then becomes a function of only six coordinates, which specify the positions of the two $D$ atoms. A convenient choice is the spherical coordinates of the center-of-mass (c.m.) of the molecule (i.e, the cluster c.m. - molecule c.m. distance and two angles; the origin of the laboratory-fixed frame is placed in the c.m. of the cluster) and the D.D bond length and orientation, specified by an additional two angles. For any fixed values of the pair of distance coordinates, one can find the minimum of the LEPS interaction energy as a function of the four angles. We have performed such minimizations on a grid of distances and angles using $0.01-0.1 \AA$ and $10^{\circ}$ as the corresponding grid intervals.

The contour map of the minimized LEPS potential energy surface is shown in Fig. 1. The entrance valley depth of $4.74 \mathrm{eV}$ at large $\mathrm{D}_{2}$ (c.m.)-Ni $\mathrm{N}_{13}$ (c.m.) distances corresponds to the well depth of the Morse potential describing an isolated $\mathrm{D}_{2}$ molecule. As the distance between the $\mathrm{D}_{2}$ and the $\mathrm{Ni}_{13}$ decreases, a shallow well corresponding to the state of molecular adsorption develops. In fact, this well represents two different adsorption configurations that have the same energy: the molecule is aligned parallel to a threefold face of the cluster and is either parallel or perpendicular to one of the edges of that face. In order to reach the barrier (saddle) for the dissociative adsorption, the molecule has to approach the cluster even closer. It is seen in Fig. 1 that the equipotential contour that corresponds to $4.55 \mathrm{eV}$ (the value of the zero-point energy of the $D_{2}$ molecule) does not reach the dissociation barrier either along the $x$-axis (D-D distance) or along the $y$-axis (clustermolecule distance). This explains why a certain minimum (threshold) collision energy is required for the molecule to be able to dissociate on the cluster (see Fig. 2). Although the barrier is a late one (it is located in the exit valley), crossing it involves both an increase in the D-D distance and a decrease in the $D_{2}$ (c.m.)-Ni 13 (c.m.) separation. This is why the reaction is enhanced not only by an initial vibrational excitation of the molecule but also by an increase of the collision energy. Five wells of approximately equal depth separated by barriers (saddles) of approximately equal height correspond to large D-D distances. These wells represent the five topologically different pairs of threefold faces of the cluster on which the molecule can adsorb dissociatively. The one with the largest D-D separation corresponds to $D$ atoms attached to opposite sides of the cluster. The most favorable atomic binding sites are the (threefold) faces. All the barriers, including that for the dissociation of the molecule, correspond to one of the $D$ atoms being over an edge between two faces. The calculation from this potential energy of binding of a D atom to the cluster is $2.43 \mathrm{eV}$. The experimental value for binding a $D$ atom to the (111) and (100) surfaces of the bulk nickel is $2.74 \mathrm{eV}$ [16]. Electronic structure calculations [3] reproduce this latter value quite well. The rate constant of 


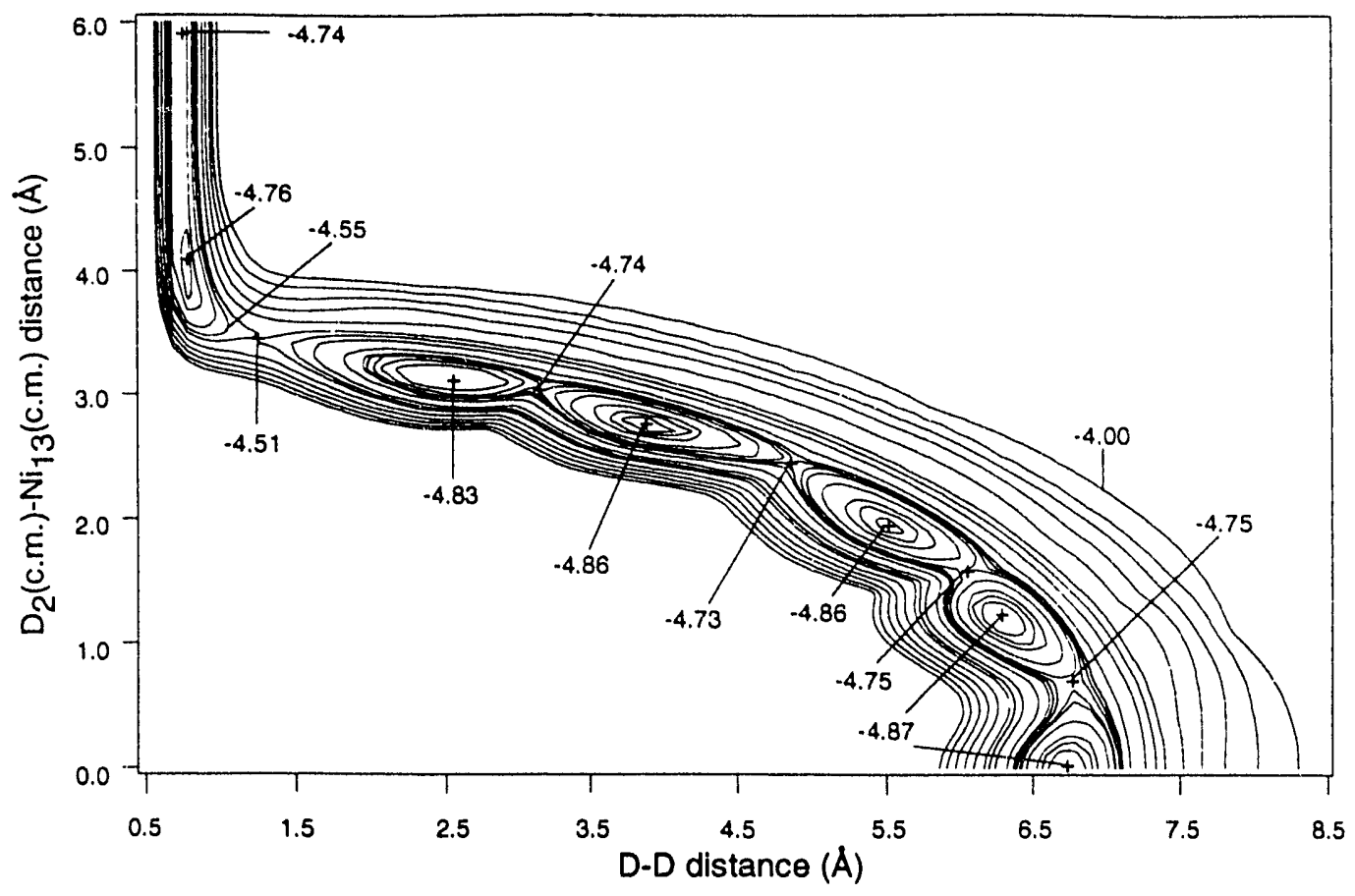

Fig. 1 Equipotential contour plot of the RSD's PESII form of the minimized (see text) LEPS potential. The crosses indicate the minima and maxima on the surface. The numbers are values of the energy in $\mathrm{eV}$.

the reaction of a $D_{2}$ with an ico $\mathrm{Ni}_{13}$ computed using this potential [10] is considerably lower than the value deduced from the experiment [15]. A cause of the discrepancy between the theory and the experiment may be that the PESII parameterization of the LEPS function underestimates the strength of the cluster-molecule interaction. (Of course, this interaction depends, in general, also on the optimized geometry of the cluster.)

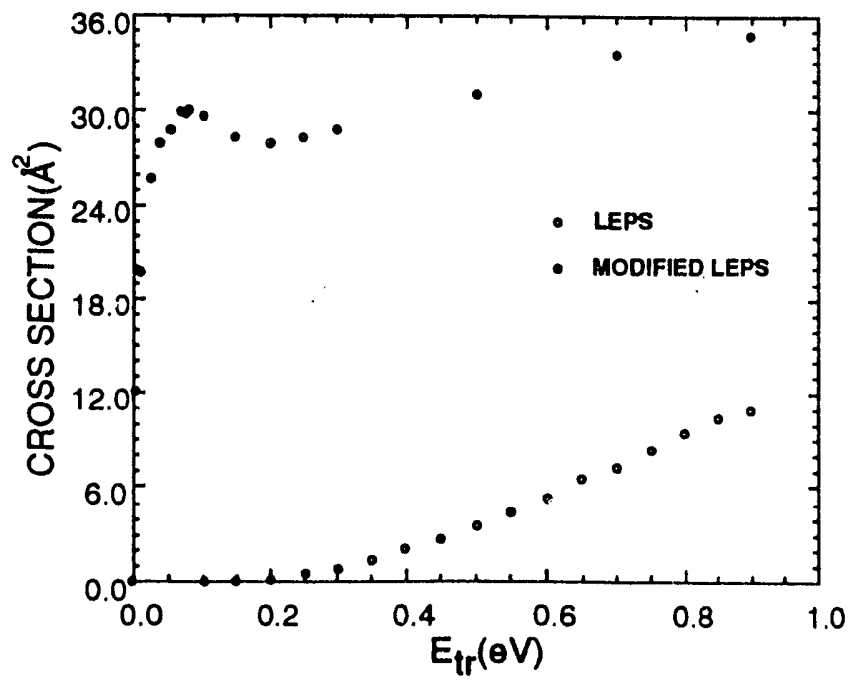

Fig. 2 Dissociative adsorption cross sections, as functions of the collision energy $E_{t}$, for the original and the modified LEPS potentials. The molecule was prepared in the vibrational $v=0$ and rotational $j=3$ state, the cluster was at room temperature.

\section{Modification of the cluster-molecule interaction energy}

To evaluate the sensitivity of the results of our dynamical simulations to the parameters of the LEPS potential and to test whether a stronger cluster-molecule interaction indeed leads to a better agreement between the theory and the experiment, we performed calculations also with a modified LEPS surface. The modified potential had the same parameters as PESII [4], except the well depths of the two Morse functions representing the $\mathrm{D}-\mathrm{Ni}$ interaction were increased by $10 \%$. This increases the $\mathrm{D}-\mathrm{Ni}_{13}$ binding energy to $2.68 \mathrm{eV}$. The ECP of the minimized over the angular degrees of freedom modified LEPS surface is shown in Fig. 3. The major difference between the plots in Figs. 1 and 3 appears in the transition region between the entrance and the exit valleys. The molecular adsorption well moves close to the exit valley. This well, however, hardly can retain the molecule, since the barrier to the dissociative adsorption is extremely low. The peak of this barrier is below not only the zero-point energy level of a $D_{2}$ but also the bottom of the entrance valley. The topology of the exit valley with the five dissociative adsorption wells remains essentially unaltered, except a $10 \%$ change in the magnitude of the corresponding energies.

It is immediately clear that the modified potential is more "reactive". It predicts that the molecule should be able to dissociate on the cluster even when its collision energy is arbitrarily small. Comparison of the reaction cross sections calculated with the original and the modified LEPS functions is showr in Fig. 2 (for computational details see Refs. $6-8,10)$. The difference between the two curves is quite striking. Not only are the cross sections computed 


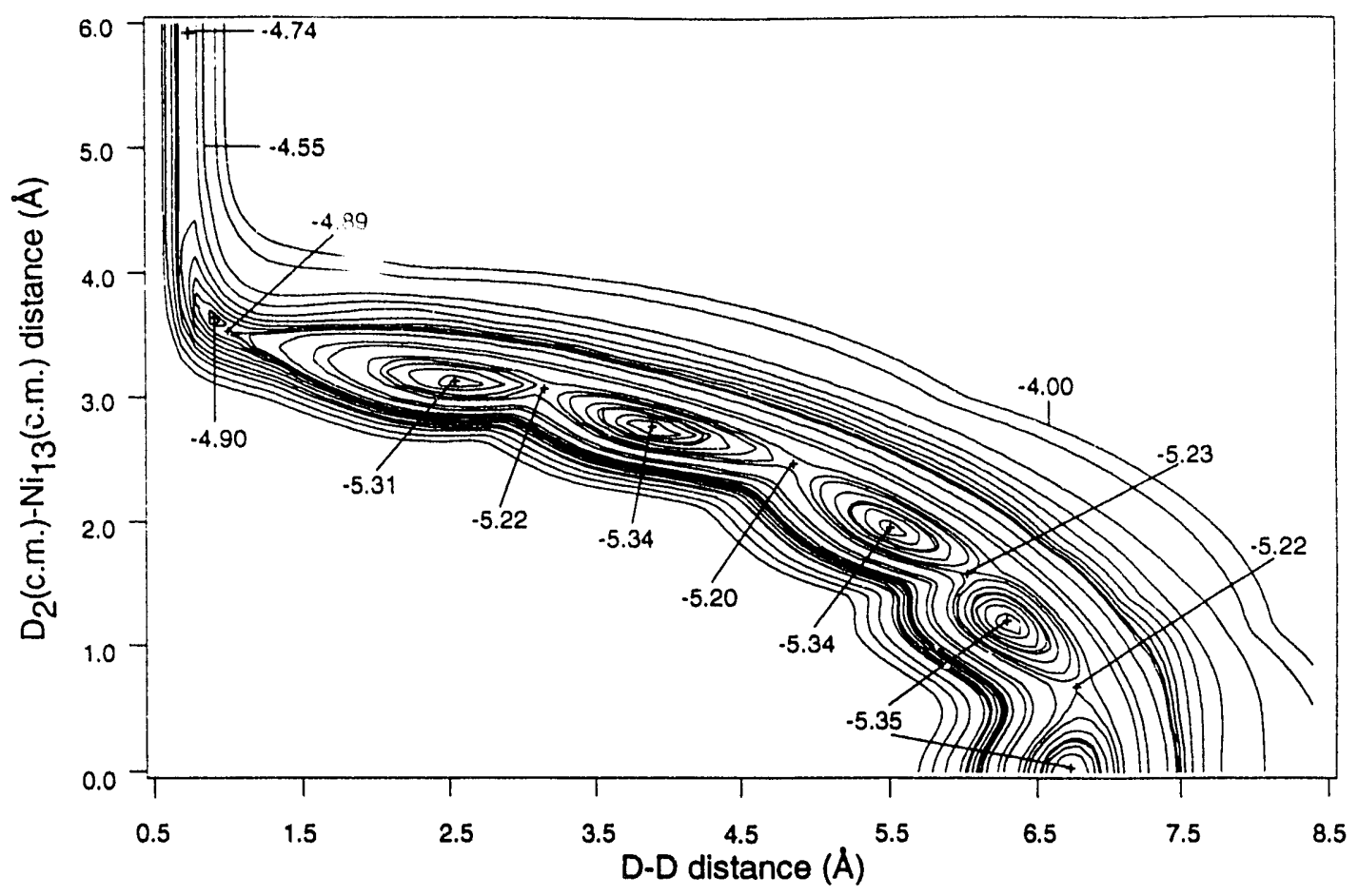

Fig. 3 The same as Fig. 1, but for the modified LEPS potential.

from the modified potential much larger, but they change nonmonotonically with the collision energy. The lowenergy maximum is a consequence of the resonance (complex formation) phenomenon $[8,10]$. It will be discussed in detail together with other aspects of the study, such as the effect of the modification of the potential on the reactivity of the hcp and cubo isomers of $\mathrm{Ni}_{13}$, elsewhere [13]. The reaction rate constant at (translational) temperature of $298 \mathrm{~K}$ computed from the cross sections corresponding to the modified potential is $3 \cdot 10^{-10} \mathrm{~cm}^{3} / \mathrm{s}$. This value is in excellent agreement with the experimental estimate of $(4 \pm 2) \cdot 10^{-10} \mathrm{~cm}^{3} / \mathrm{s}[15]$. We have calculated also the frequency of vibration of a $D$ atom adsorbed on the cluster. The original potential gives the value $754 \mathrm{~cm}^{-1}$, the modified $-791 \mathrm{~cm}^{-1}$. For an $\mathrm{H}$ atom, these frequencies translate into $1066 \mathrm{~cm}^{-1}$ and $1123 \mathrm{~cm}^{-1}$, respectively. Both values are very close to the measured $\left(1122-1137 \mathrm{~cm}^{-1}[17]\right)$ and calculated (1043-1176 cm-1 [3b]) frequencies of vibration of an $\mathrm{H}$ atom over a threefold site of $\mathrm{Ni}$ (111) surface.

\section{Summary}

An analysis of the interaction of a $D_{2}$ molecule with an ico $\mathrm{Ni}_{13}$ cluster as modelled by a LEPS potential has been presented in terms of contour plots. The ECP representation of the minimized interaction energy displays topological features that directly correlate with and explain the energetic and dynamical characteristics of the dissociative adsorption of the molecule on the cluster as obtained in our quasiclassical simulations. We have introduced a modifica- tion of the RSD's PESII form of the LEPS potential, which brings the calculated reaction rate constant into an agreement with the experimental value. Our modification is based on the presumption that the original LEPS function underestimates the strength of the cluster-molecule interaction. This presumption derives from the comparison of the $D$-cluster binding energy calculated from the original LEPS potential to the better established value of the $D$ surface binding energy. Ultimately, however, detailed enough experimental and $a b$ initio data for clusters will have to be used to tune and to test models of clustermolecule interactions.

\section{References}

1. See, for example, Elkind, J.L., Weiss, F.D., Alford, J.M., Laaksonen, R.T., Smalley, R.E.: J. Chem. Phys. 88, 5215 (1988); Reents Jr., W.D., Mandich, M.L.: J. Phys. Chem. 92, 2908 (1988); Ruatta, S.A., Anderson, S.L.: J. Chem. Phys. 89, 273 (1988); Hoffman, W.F., Parks, E.K., Riley, S.J.: J. Chem. Phys. 90, 1526 (1989); Hamrick, Y.M., Morse, M.D.: J. Phys. Chem. 93, 6494 (1989); Loh, S.K., Lian, L., Armentrout, P.B.: J. Chem. Phys. 91, 6148 (1989); Nonose, S., Sone, Y., Onodera, K., Sudo, S., Kaya, K.: Chem. Phys. Lett. 164, 427 (1989); Fayet, P., Kaldor, A., Cox, D.M.: ibid. 92, 254 (1990); Creegan, K.M., Jarrold, M.F.: J. Am. Chem. Soc. 112, 3768 (1990); Leuchtner, R.E., Harms, A.C., Casulemean Jr., A.W.: J. Chem. Phys. 92, 6527 (1990); Irion, M.P., Schnabel, P.: J. Phys. Chem. 95, 
10596 (1991); Knickelbein, M.B., Menezes, W.J.C.: J. Phys. Chem. 96, 6611 (1992); and references therein

2. See, for example, Upton, T.H.: Phys. Rev. Lett. 56, 2168 (1986); Upton, T.H., Cox, D.M., Kaldor, A.: In: Physics and Chemistry of Small Clusters. Jena, P., Rao, B.K., Khanna, S.N. (eds.). New York: Plenum Press, 1987, p. 755; Przybylski, K., Koutecký, J., Bonacic-Koutecký, V., von Ragué-Schleyer, P., Guest, M.F.: J. Chem. Phys. 94, 5533 (1991); Mijoule, C., Bouteiller, Y., Salahub, D.R.: Surf. Sci. 253, 375 (1991); and references therein

3. See, for example, (a) Siegbahn, P.E.M., Blomberg, M.R.A., Bauschlicher, C.W.: J. Chem. Phys. 81, 2103 (1984); Panas, I., Schüle, J., Siegbahn, P., Wahlgren, U.: Chem. Phys. Lett. 149, 265 (1988); (b) Yang, H., Whitten, J.L.: J. Chem. Phys. 89, 5329 (1988); (c) Mlynarski, P., Salahub, D.R.: J. Chem. Phys. 95, 6050 (1991); and references therein

4. Raghavan, K., Stave, M.S., DePristo, A.E.: Chem. Phys. Lett. 149, 89 (1988); J. Chem. Phys. 91, 1904 (1989)

5. Adams, J.E.: J. Chem. Phys. 92,1849 (1990)

6. Jellinek, J., Güvenç, Z.B.: Z. Phys. D - Atoms, Molecules and Clusters 19, 371 (1991)

7. Jellinek, J. Güvenç, Z.B.: In: Mode Selective Chemistry. Jortner, J., Levine, R.D., Pullmann, B. (eds.). Dordrecht: Kluwer Academic Publishers, 1991, p. 153

8. Jellinek, J., Güvenç, Z.B.: In: Physics and Chemistry of Finite Systems: From Clusters to Crystals, vol. II. Jena, P., Rao, B.K., Khanna, S.N. (eds.). Dordrecht: Kluwer Academic Publishers, 1992, p. 1047

9. Fournier, R., Stave, M.S., DePristo, A.: J. Chem. Phys. 96, 1530 (1992)

10. Jellinek, J. Güvenç, Z.B.: In: Nuclear Physics Concepts in Atomic Cluster Physics, Lecture Notes in Physics. Lutz, H.O., Schmidt, R., Dreizler, R. (eds.). Heidelberg: Springer-Verlag (in press)

11. Voter, A.F., Chen, S.F.: Mater. Res. Soc. Symp. 82, 175 (1987)

12. Truong, T.N., Truhlar, D.G., Garett, B.C.: J. Phys. Chem. 93, 8227 (1989)

13. Güvenç, Z.B., Jellinek, J.: to be puolished

14. Zhu, L., Ho, J., Parks, E.K., Riley, S.J.: to be published

15. Hoffman, W.F., Parks, E.K., Nieman, G.C., Pobo, L.G., Riley, S.J.: Z. Phys. D - Atoms, Molecules and Clusters 7, 83 (1987)

16. Christmann, K., Schober, O., Ertl, G., Neuman, M.: J. Chem. Phys. 60, 4528 (1974)

17. Ho, W., DiNardo, N. J., Plummer, D.E.: J. Vac. Sci. Technol. 17, 314 (1980); Cavanagh, R.R., Kelley, R.D., Rush, J.J.: J. Chem. Phys. 77, 1540 (1982) 

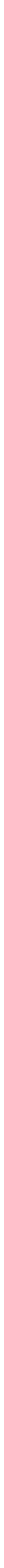
